\title{
Elaboração de modelo de maturidade (Indústria 4.0) para fabricantes de componentes industrializados
}

\author{
Development of a maturity model (Industry 4.0) for \\ industrialized component manufacturers
}

\section{Cayami Schultz Chiovitti Cavalcante}

Universidade de São Paulo | São Paulo| Brasil | cayami@alumni.usp.br

Fabiano Rogerio Corrêa

Universidade de São Paulo | São Paulo| Brasil | fabiano.correa@usp.br

\section{Resumo}

A Indústria 4.0 visa otimizar operações em tempo real e criar modelos de negócios completamente inovadores pela introdução de tecnologias como Internet das Coisas e Sistemas Ciberfísicos no ambiente industrial. Para que fabricantes de componentes para sistemas construtivos industrializados se beneficiem dessa revolução industrial, é necessário contextualizar o conceito da Indústria 4.0 para o setor da construção pré-fabricada. Como parte de uma pesquisa de mestrado em progresso, esse artigo utiliza uma abordagem de Design Science Research para sistematizar as tecnologias da Indústria 4.0, propor uma configuração da Indústria 4.0 para fabricantes de componentes para sistemas construtivos e desenvolver uma ferramenta de avaliação de maturidade para caracterizar o estágio atual de desenvolvimento tecnológico do setor segundo conceitos da Indústria 4.0.

Palavras-chave: Indústria 4.0. Sistemas construtivos. Pré-fabricação. Industrialização.

\begin{abstract}
Industry 4.0 aims to optimize operations in real time and create innovative business models by introducing technologies such as Internet of Things and Cyberphysical Systems in the industrial environment. In order for manufacturers of components for industrialized building systems to benefit from this industrial revolution, it is necessary to contextualize the concept of Industry 4.0 for the prefabricated construction sector. As part of a master's thesis in-progress, this paper uses a Design Science Research approach to systematize the technologies of Industry 4.0, propose an Industry 4.0 configuration for building component manufacturers and develop a maturity assessment tool in order to characterize the current stage of technological development of the sector according to Industry 4.0 concepts.
\end{abstract}

Keywords: Industry 4.0. Construction systems. Prefabrication. Industrialization.

\section{INTRODUÇÃO}

A Industrialização da construção é um processo evolutivo que envolve implementação de inovações tecnológicas, além de novos métodos de trabalho e outras ações organizacionais, com objetivo de aumentar a capacidade de transformação de recursos em produtos [1].

Como citar:

CAVALCANTE, C. S. C.; CORRÊA, F. R. Elaboração de modelo de maturidade (Indústria 4.0) para fabricantes de componentes industrializados. In: SIMPÓSIO BRASILEIRO DE TECNOLOGIA DA INFORMAÇÃO E COMUNICAÇÃO NA CONSTRUÇÃO, 3., 2021, Uberlândia. Anais [...]. Porto Alegre: ANTAC, 2021. p. 1-13. Disponível em:

https://eventos.antac.org.br/index.php/sbtic/article/view/569. Acesso em: 3 ago. 2021. 
A pré-fabricação de componentes exerce papel importante na industrialização da construção e significa a produção de produtos construtivos através de técnicas industriais em plantas especializadas onde elementos podem ser manufaturados independentemente de clima e debaixo de condições ótimas de produção [2]. Técnicas industriais incluem não somente ferramentas e tecnologias para mecanização e automação de processos, mas também a aplicabilidade de ciências de administração da produção industrial como Fordismo, Lean, entre outros. Do contexto controlado sucede a redução de incertezas que apresentam riscos ao planejamento da produção. A repetibilidade de processos permite a eliminação de desperdícios e otimização operacional resultando, por fim, em vantagens como qualidade constante e a redução de prazos e custos de construção.

Sistemas construtivos utilizam componentes pré-fabricados com elevados níveis de industrialização e organização [1]. A pré-fabricação industrial de componentes para sistemas construtivos surgiu junto com a primeira revolução industrial no século XIX, com a produção serial de elementos estruturais em aço [2]. Outros exemplos de sistemas construtivos são: concreto pré-moldado/protendido, light steel frame, wood frame e madeira laminada cruzada.

Apesar da pré-fabricação industrial para construção não ser um conceito novo, há uma renovação recente de interesses tanto da academia quanto da indústria [3][4] devido aos benefícios potenciais (redução de prazos e custos de construção), a ampla adoção de tecnologias digitais como ferramentas de Modelagem de Informação de Construção (BIM) por profissionais da construção e a crescente conscientização sobre a construção sustentável [3]. De acordo com [4], as tecnologias digitais aumentam a qualidade e variabilidade do projeto, melhoram a precisão e a produtividade no processo de fabricação e ainda facilitam a coordenação logística entre agentes da cadeia de valor.

O termo "Indústria 4.0" (14.0) se refere ao quarto estágio da revolução industrial na manufatura, que se desenvolve a partir dos três primeiros: 11.0- mecanização; 12.0emprego de eletricidade e linhas de produção; 13.0- tecnologias de informação e comunicação. A 14.0 será proporcionada pela convergência de novas tecnologias, promovendo um "processo de transformação na indústria além da automação, envolvendo monitoramento inteligente e processos de tomada de decisão autônomos através da Internet das Coisas (IOT) baseada em Sistemas Ciberfísicos (CPS)" [5]. A visão é de atingir a capacidade de otimizar logística e produção de redes de valor completas em tempo real e ainda 'criar modelos de negócios inovadores mais focados em atendimento de requisitos individuais customizados de forma ágil' [6].

Devido ao ambiente estruturado da fábrica, a 14.0 apresenta potenciais aplicações para Fabricantes de Componentes para Sistemas Construtivos Industrializados (FSCls) permitindo abordar simultaneamente questões de produtividade dos processos de produção e variabilidade dos projetos de edificações.

Existe uma lacuna na literatura acadêmica e técnica de uma configuração sistematizada da 14.0 na construção civil, com o objetivo de orientar o seu desenvolvimento tecnológico e industrial. Especificidades de projeto e produção de 
componentes, e desafios próprios da indústria da construção denotam a necessidade de uma abordagem específica para direcionar ações estratégicas organizacionais.

É necessário conhecer o estágio atual de desenvolvimento tecnológico de $\mathrm{FSCls}$ segundo tecnologias da 14.0. Para tanto, esta pesquisa, tem por objetivo desenvolver o Modelo de Maturidade da Indústria 4.0 para FSCls (MM-FSCI4.0), uma ferramenta de diagnóstico para fabricantes individuais associada a um sistema de pontuação para determinar o seu Índice de Maturidade (IM-4.0).

O MM-FSCl4.0 servirá tanto para a caracterização individual das empresas quanto para a informar o quadro atual do setor. A avaliação da maturidade dos $\mathrm{FSCls} \mathrm{é} \mathrm{um} \mathrm{ponto}$ de partida importante para a realização efetiva da 14.0 no setor da construção.

Os objetivos específicos envolvem:

1. Sistematizar o escopo tecnológico da 14.0, as inter-relações entre as tecnologias e pré-requisitos para sua implantação;

2. Propor uma configuração das tecnologias da 14.0 para $\mathrm{FSCls}$ a partir do conjunto de tecnologias sistematizadas para a 14.0;

3. Projetar o MM-FSCI4.0.

\section{MÉTODO}

O método utilizado é a Design Science Research, "abordagem metodológica prescritiva orientada à solução de problemas específicos do mundo real, por meio da construção e avaliação de artefatos [7], conforme Figura 1.

É utilizado o método sistêmico para apoiar a solução da projeção de desenvolvimento de tecnologias industriais e as inter-relações entre essas tecnologias no contexto dos $\mathrm{FSCls}$.

A partir da identificação do problema, a pesquisa divide a conscientização do mesmo em duas etapas.

1) Sistematização das tecnologias da 14.0:

a. Delimitação do escopo pela identificação das tecnologias-chave da 14.0. Envolve consulta a revisões sistemáticas e publicações técnicas mais citadas no tema;

b. Definições das tecnologias identificadas e discernimento dos prérequisitos.

2) Configuração tecnológica da 14.0 para os $\mathrm{FSCls:}$

a. Identificação e análise das tecnologias específicas da Indústria da Construção que representam o sentido de desenvolvimento do setor que demonstram potencial para confluir com a concepção da 14.0. Aqui é realizada uma revisão sistemática da literatura, investigando a ocorrência simultânea das palavras-chave das tecnologias da 14.0 com a pré-fabricação ou construção pré-fabricada no tópico de artigos científicos utilizando a base da Web of Science.

b. Estruturação das inter-relações entre as tecnologias identificadas. 
O desenvolvimento do $\mathrm{MM}-\mathrm{FSCl} 4.0$ parte da conscientização do problema para estabelecer o escopo de análise e requisitos. É importante avaliar a aplicabilidade de modelos/índices de maturidade existentes de outras indústrias. Artefatos desenvolvidos para resolver problemas similares, especificamente voltados para Indústria 4.0 ou para BIM, fornecem uma 'forma de assegurar que a pesquisa que está sendo desenvolvida oferece uma contribuição relevante' [7]. A análise de aplicabilidade dos modelos preexistentes inclui o atendimento do escopo de análise e requisitos, e a identificação de lacunas. A partir desse embasamento, propõem-se um projeto conceitual para o MM-FSCI4.0, as perguntas e as escalas de respostas.

Figura 1: Metodologia de pesquisa para o desenvolvimento do modelo de maturidade

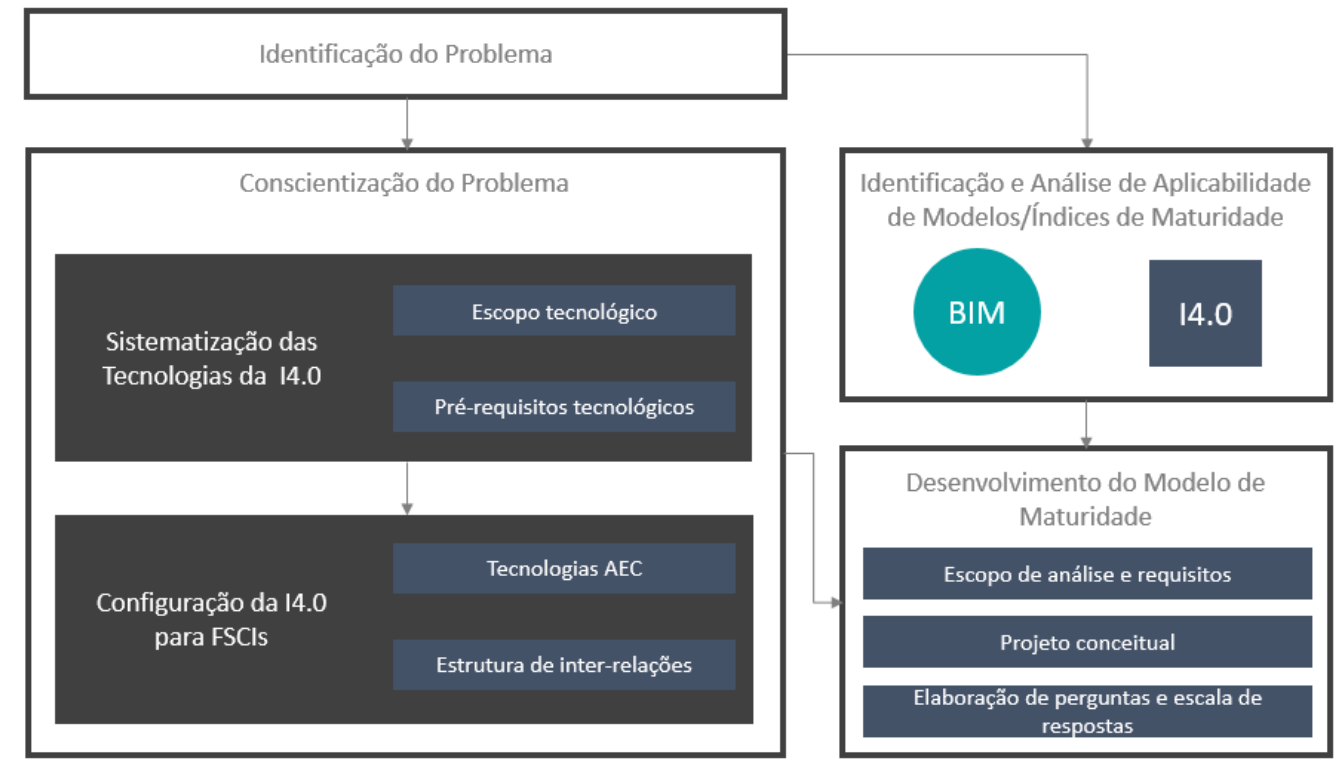

Fonte: os autores.

\section{SISTEMATIZAÇÃO DAS TECNOLOGIS DA I4.0 E CONFIGURAÇÃO PARA FSCI}

A avaliação de revisões sistemáticas da 14.0 que utilizam métodos diferentes e exploram o tema com enfoques distintos permite uma compreensão abrangente da temática abordada. É possível identificar e priorizar tecnologias em diferentes níveis desde implementações específicas até níveis mais altos de abstrações de conceitos e temas de pesquisa. Com esta síntese de resultados, é fornecida uma resposta concreta à delimitação do escopo da 14.0 e são estabelecidas prioridades para composição de tecnologias que poderão ser implementadas no contexto das pré-fabricação industrial para a construção. A Figura 2 demonstra as inter-relações entre as tecnologias dominantes na literatura acadêmica referente à 14.0, e a sua configuração dessas tecnologias para os $\mathrm{FSCls}$. 
Figura 2: Estrutura de relações entre tecnologias da 14.0 para $\mathrm{FSCls}$

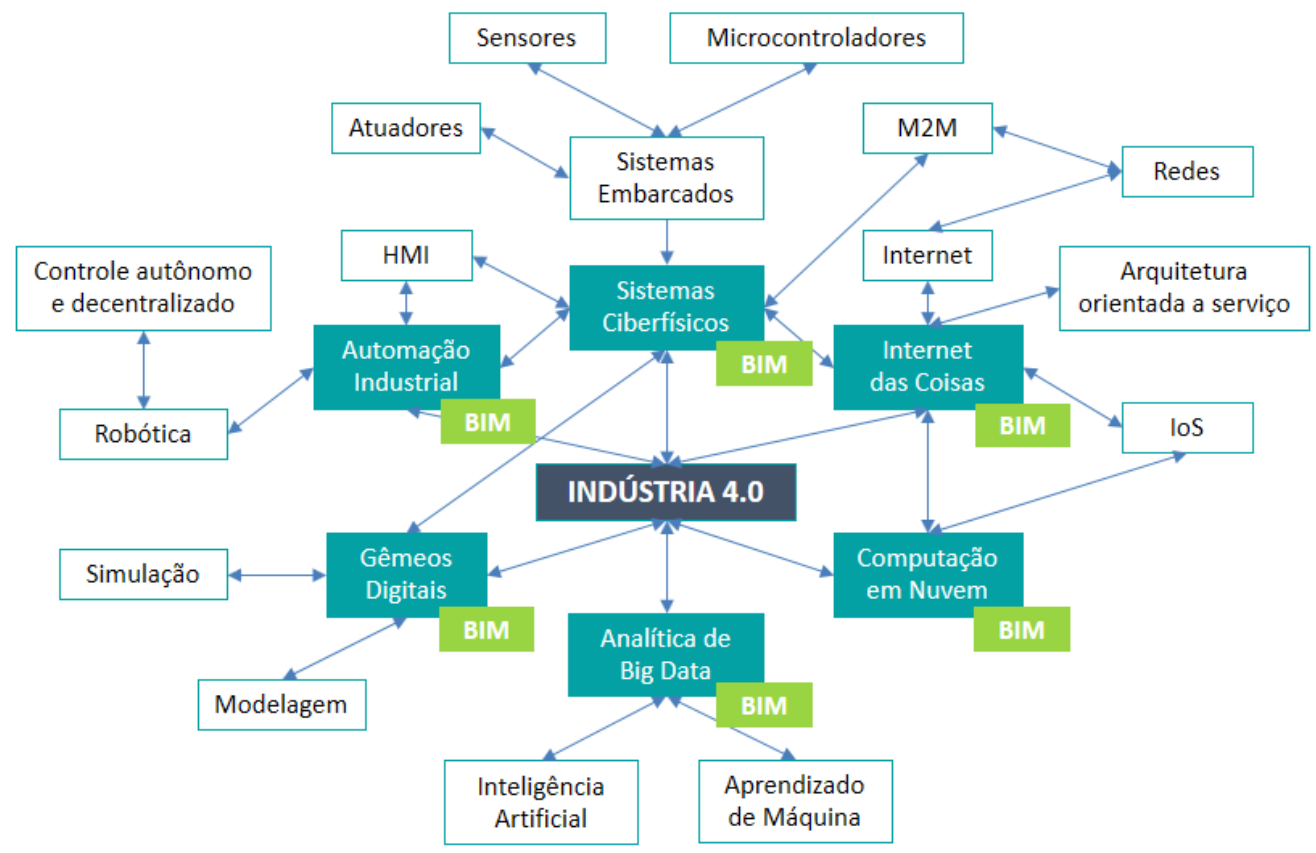

Fonte: os autores.

\section{MODELAGEM DA INFORMAÇÃO DA CONSTRUÇÃO}

Modelagem da Informação da Construção (BIM) é um processo integrado para criar, usar e atualizar um modelo digital de uma construção, podendo ser usado por todos os participantes do empreendimento, potencialmente durante todo o ciclo de vida da construção [8]. A presença do BIM é ubíqua na digitalização na indústria da construção, possuindo interface com as demais tecnologias mais relevantes à 14.0. BIM e préfabricação são dois paradigmas reivindicados para potencialmente resolver os problemas de eficiência e produtividade na indústria da construção e promover inovações profundas [9]. BIM para a pré-fabricação possibilita design aprimorado, facilita a colaboração e cobre uma quantidade extensa e precisa de informações [10].

\section{AUTOMAÇÃO INDUSTRIAL E ROBÓTICA}

Automação Industrial e Robótica são tecnologias principais da terceira revolução industrial, pré-requisitos para a 14.0. O aumento da automação é uma consequência lógica da digitalização [11]. FSCls podem utilizar a entrada de dados paramétricos de objetos BIM (não fornecidos pelo CAD-2D convencional) para auxiliar o processo de fabricação [12], inclusive manufatura digital através de controle numérico computadorizado (CNC) e impressão 3D.

Na construção atual, os sistemas de manufatura automatizados são usados quase exclusivamente no contexto da pré-fabricação [11]. Orlowski [13] explora os processos na fabricação automatizada em painéis de madeira que tem mostrado níveis crescentes de automação.

$\mathrm{Na}$ fabricação de estruturas de concreto pré-moldado, a circulação de paletes começam a substituir as fôrmas fixas; um passo para automação e robótica [14]. Junto com Projeto e Manufatura Assistidos por Computadores (CAD/CAM) e introdução de 
Controladores Lógicos Programáveis (PLCS), máquinas automatizadas e robóticas foram introduzidas, como plotters, robôs de fôrma e espalhadores de concreto automatizados [15].

A construção em aço é caracterizada por um alto grau de pré-fabricação em comparação com outras tipologias construtivas [11]. Sistemas especializados executam serração, furação e soldagem em processos repetitivos.

Avançando para 14.0, esperam-se ver instalações de produção mais flexíveis através de robótica e manufatura aditiva, padronização de interfaces CAD/CAM, interfaces operacionais mais intuitivas e agregação de ciência de contexto.

\section{SISTEMAS CIBERFÍSICOS}

Os Sistemas Ciberfísicos (CPS) são prioritários ao 14.0 desde sua concepção inicial. Geisberger et al. [16] identificam múltiplas formas pelas quais os CPS agregam valor para fábricas inteligentes, incluindo otimização de processos de produção e fabricação de produtos individualizados.

CPS são integrações de computação com processos físicos [17]. Computadores e redes incorporados monitoram e controlam os processos físicos, geralmente com loops de feedback, onde os processos físicos afetam os cálculos e vice-versa. Tradicionalmente, os sistemas embarcados eram vistos simplesmente como pequenos computadores com processamento e memória limitados. A engenharia desses sistemas tinha foco na otimização [18]. No entanto, devido à crescente integração e sinergia de objetos físicos e processos computacionais, o termo CPS foi introduzido [19].

\section{INTERNET DAS COISAS}

A Internet das Coisas (IoT) pode ser apresentada como mais uma fase de desenvolvimento e evolução da internet e de componentes de rede [20]. A loT descreve computadores capazes de coletar informações por si mesmos, sem necessidade de entrada por parte de pessoas usando teclados. O compartilhamento dessas informações pela internet e entre máquinas (M2M) permite uma enorme variedade de novas aplicações, incluindo produtos e serviços inteligentes. $O$ uso crescente da loT na manufatura transformará as cadeias de valor e levará ao surgimento de novos modelos de negócios [6].

A integração de BIM e IoT possibilita o monitoramento, compartilhamento e a comunicação de informações em tempo real, como dados ambientais, localização de componentes e equipamentos, progresso e produtividade do trabalho, que auxiliam as operações de construção e gerenciamento [21]. Essa integração é um passo significativo na direção da automação para pré-fabricação. Informações de tags RFID anexas a componentes pré-fabricados podem ser integradas com modelos BIM para rastreamento, localização, visualização facilitada e montagem automática.

\section{ANALÍTICA DE BIG DATA (ABD)}

No ambiente industrial, uma riqueza de dados provenientes de sensores de diversos tipos está disponível e cria grandes oportunidades para gerar melhor compreensão, gestão e exploração estratégica através da analítica de dados. Big Data compreende 
dados que não podem ser tratados com software tradicional e que nem mesmo poderiam ser armazenados em uma única máquina, tradicionalmente caracterizados por seu Volume, Velocidade e Variedade.

\section{COMPUTAÇÃO EM NUVEM}

Hoje, grande parte dos processos de fabricação industrial já são suportados por Tecnologias da Informação e Comunicação (TICS) e existe uma tendência de cada vez mais infraestrutura e serviços de TIC migrarem para a Computação em Nuvem (CN) que permite acesso onipresente, conveniente e sob demanda por rede a um conjunto compartilhado de recursos (redes, servidores, armazenamento, aplicativos e serviços) [22].

Reconhecida como um paradigma para armazenamento e análise de big data [28], das interconexões entre objetos inteligentes e computação quase omnipresente, se manifesta um mundo ciberfísico para o qual a Computação em Nuvem providencia parte crucial da infraestrutura necessária.

\section{GÊMEOS DIGITAIS}

GD é um constructo digital de informações sobre um sistema físico [24], incluindo modelos baseados em física quanto modelos baseados em dados de sensores [25], permitindo otimização em tempo real e simulação de cenários futuros [26]. O GD espelha todo o ciclo de vida de seu gêmeo físico [27] (ativos ou produtos), agindo como fonte única de verdade [28]. Associado à integração ciberfísica [29], o GD cria um canal bidirecional através do qual os mundos físico e digital se influenciam.

\section{DESENVOLVIMENTO DO MODELO DE MATURIDADE}

O Quadro 1 apresenta uma lista não exaustiva de modelos/índices de maturidade da I4.0 e BIM. Nota-se claramente uma lacuna de abordagem do processo de projeto nos modelos/índices da 14.0. Como é de se esperar, não há tentativa de modelos/índices BIM ou VDC (Virtual Design and Construction) de abordar a integração do projeto e da produção automatizada. Isso indica a necessidade de uma ferramenta de avaliação projetada especificamente para $\mathrm{FSCls}$.

Quadro 1: Análises de Aplicabilidade de Modelos de Maturidade

\begin{tabular}{|c|c|c|c|c|c|c|c|c|c|c|}
\cline { 2 - 12 } \multicolumn{1}{c|}{} & \multicolumn{7}{c|}{ Tecnologias-chave } & \multicolumn{4}{c|}{ Escopo de interesse } \\
\hline PAPER & BIM & AUTO & CPS & IOT & CN & ABD & CD & Projeto & Produção & $\begin{array}{c}\text { Logística } \\
\text { Interna }\end{array}$ \\
\hline$[30]$ & x & $\checkmark$ & x & $\checkmark$ & $\checkmark$ & $\checkmark$ & x & x & $\checkmark$ & $\checkmark$ \\
\hline$[31]$ & $\mathrm{x}$ & $\checkmark$ & $\mathrm{x}$ & $\checkmark$ & $\checkmark$ & $\checkmark$ & $\mathrm{x}$ & $\mathrm{x}$ & $\checkmark$ & $\checkmark$ \\
\hline$[32]$ & $\mathrm{x}$ & $\checkmark$ & $\checkmark$ & $\checkmark$ & $\checkmark$ & $\checkmark$ & $\checkmark$ & $\mathrm{x}$ & $\checkmark$ & $\checkmark$ \\
\hline$[33]$ & $\checkmark$ & $\checkmark$ & $\checkmark$ & $\checkmark$ & $\checkmark$ & $\checkmark$ & $\checkmark$ & $\mathrm{x}$ & $\checkmark$ & $\checkmark$ \\
\hline$[34]$ & $\mathrm{x}$ & $\checkmark$ & $\checkmark$ & $\checkmark$ & $\checkmark$ & $\checkmark$ & $\checkmark$ & $\mathrm{x}$ & $\checkmark$ & $\checkmark$ \\
\hline$[35]$ & $\mathrm{x}$ & $\checkmark$ & $\checkmark$ & $\checkmark$ & $\checkmark$ & $\checkmark$ & $\mathrm{x}$ & $\mathrm{x}$ & $\checkmark$ & $\checkmark$ \\
\hline$[36]$ & $\checkmark$ & $\mathrm{x}$ & $\mathrm{x}$ & $\mathrm{x}$ & $\mathrm{x}$ & $\mathrm{x}$ & $\mathrm{x}$ & $\checkmark$ & $\mathrm{x}$ & $\mathrm{x}$ \\
\hline$[37]$ & $\checkmark$ & $\mathrm{x}$ & $\mathrm{x}$ & $\mathrm{x}$ & $\mathrm{x}$ & $\mathrm{x}$ & $\mathrm{x}$ & $\checkmark$ & $\mathrm{x}$ & $\mathrm{x}$ \\
\hline
\end{tabular}

Fonte: os autores. 


\section{MODELO DE MATURIDADE PROPOSTO}

O objetivo geral do $\mathrm{MM}-\mathrm{FSCl} 4.0$ é fornecer um meio de determinar o estágio de maturidade tecnológico-industrial atual das empresas $\mathrm{FSCls}$, com foco no conjunto de tecnologias que, através de avaliação sistemática da literatura, demonstram prioridade para o desenvolvimento no futuro próximo.

Para esse estudo, o escopo de interesse é no projeto e produção de componentes para sistemas construtivos industrializados projetados sob demanda incluindo atividades de logística interna. Uma sequência esquemática abstraída do processo na fábrica é inviável devido à diversidade de processos produtivos entre fabricantes de diferentes tipos (e.g., concreto pré-moldado, estruturas metálicas ou wood frame). Optou-se, ao invés disso, por uma análise de alto nível descrita por dois artefatos de apoio: (1) Modelo de interações entre o $\mathrm{FSCl}$ com fornecedores e clientes, conforme indicado na Figura 3; (2) Dimensões de análise e processos constituintes na Figura 4.

Figura 3: Diagrama de interações entre agentes analisados no MM-FSCl4.0

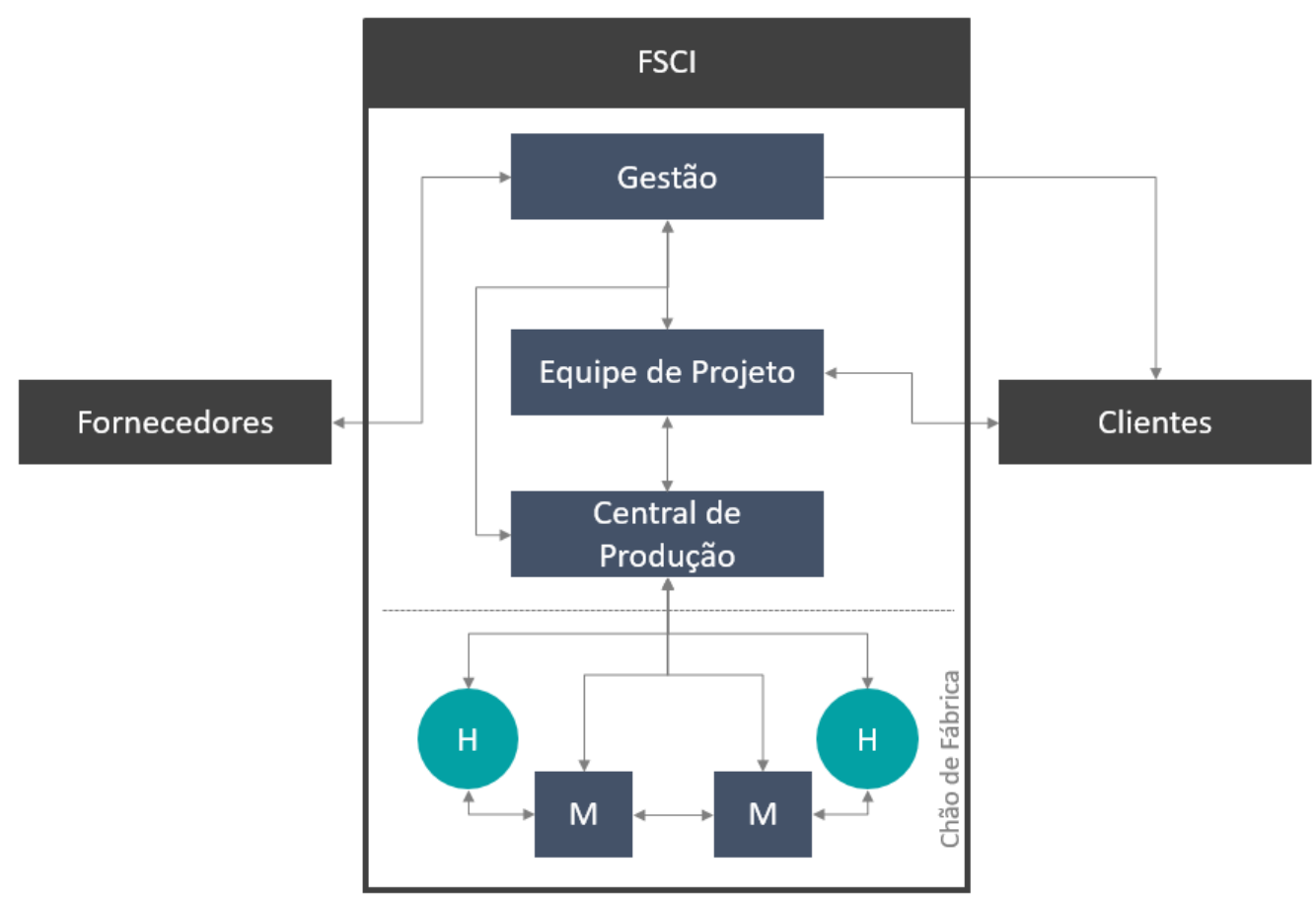

Fonte: os autores.

O Diagrama da Figura 3 apresenta o $\mathrm{FSCl}$ como figurante central da análise. Internamente dividido em gestão, equipe de projeto, central e produção e chão de fábrica com máquinas e humanos. Inclui ainda aspectos das interações com os fornecedores e clientes mais próximos ao escopo de projeto e produção.

O MM-FSCl4.0 considera a existência de duas dimensões entrelaçadas, mas distintas: Dimensão Virtual e Dimensão Física. Essa descrição está em linha com o uso do conceito de CPS no contexto da 14.0. Na Dimensão Virtual estão incluídos todos os processos que envolvem fluxo de dados e informações: (i) Desenvolvimento de Projeto; (ii) Entrada de parâmetros de produção; (iii) Sensoriamento; (iv) Transmissão de dados; (v) Armazenamento e processamento de dados; (vi) Aplicações Avançadas. 
A Dimensão Física inclui os processos que envolvem fluxo material: Produção e Logística Interna. Veja Figura 4.

O modelo apresenta 30 questões, cada uma associada a uma Dimensão Física e/ou uma Dimensão Virtual e também a uma tecnologia-chave da 14.0 ou pré-requisito tecnológico. Cada questão possui uma escala de cinco respostas possíveis ao longo de uma escala crescente de nível de desenvolvimento associada diretamente à escala de pontuação máxima, exemplificado pelos Quadro 2 e Quadro 3. O IM-4.0 é dado pela média aritmética das pontuações nas 30 questões do modelo.

Figura 4: Dimensões de análise e processos constituintes

\section{DIMENSÃO VIRTUAL \\ (FLUXO DE DADOS E INFORMAÇÕES)}

Desenvolvimento de Projeto

Entrada de parâmetros de produção

Sensoriamento

Transmissão de dados

Armazenamento e processamento de dados

Aplicações avançadas
DIMENSÃO FÍSICA

(FLUXO MATERIAL)

Produção

Logística

Fonte: os autores.

Quadro 2: Primeiro exemplo de questão do MM-FSCI4.0

\begin{tabular}{|l|l|}
\hline Dimensão Física & \\
\hline Dimensão Virtual & Desenvolvimento de Projeto \\
\hline Tecnologia/Conceito & Software de Projeto: CAD/BIM/CAM \\
\hline Questão & Como se caracterizam os softwares de desenvolvimento de projetos em sua empresa? \\
\hline max pts. 0 & $\begin{array}{l}\text { Trabalhos com projetos 2D desenvolvidos em AutoCAD ou outro software CAD } \\
\text { equivalente. }\end{array}$ \\
\hline max pts. 25 & $\begin{array}{l}\text { Trabalhamos com projetos 2D desenvolvidos em AutoCAD ou outro software CAD } \\
\text { equivalente. Utilizamos outros softwares de modelagem 3D não-semânticos como } \\
\text { Skecthup para auxiliar na visualização. }\end{array}$ \\
\hline max pts. 50 & $\begin{array}{l}\text { Trabalhamos com ferramentas BIM apropriadas para nossos processos de produção. } \\
\text { As ferramentas são capazes de gerar processos executivos para produção em fábrica. }\end{array}$ \\
\hline max pts. 75 & $\begin{array}{l}\text { Trabalhamos com ferramentas BIM apropriadas para nossos processos de produção. } \\
\text { As ferramentas são capazes de gerar processos executivos para produção em fábrica. }\end{array}$ \\
\hline max pts. 100 & $\begin{array}{l}\text { Trabalhamos com ferramentas BIM apropriadas para nossos processos de produção. } \\
\text { As ferramentas são capazes de gerar processos executivos para produção em fábrica } \\
\text { e gerar instruções computacionais necessárias para operação do maquinário. }\end{array}$ \\
\hline
\end{tabular}

Fonte: os autores. 
Quadro 3: Segundo exemplo de questão do MM-FSCl4.0

\begin{tabular}{|l|l|}
\hline Dimensão Física & Produção \\
\hline Dimensão Virtual & Aplicações avançadas \\
\hline Tecnologia/Conceito & CPS (M2M). Sistemas flexíveis, reconfiguráveis, autônomos. \\
\hline Questão & $\begin{array}{l}\text { A empresa possui máquinas capazes de ajustar sua operação de acordo com } \\
\text { informações recebidas de outras máquinas? }\end{array}$ \\
\hline max pts. 0 & Não. \\
\hline max pts. 25 & $\begin{array}{l}\text { Sim. Já fizemos experimentos com máquinas com capacidade de ajustar a operação de } \\
\text { acordo com informações recebidas de outras máquinas. }\end{array}$ \\
\hline max pts. 50 & $\begin{array}{l}\text { Sim. Temos algumas máquinas com capacidade de ajustar a operação de acordo com } \\
\text { informações recebidas de outras máquinas (até 25\% das máquinas). }\end{array}$ \\
\hline max pts. 75 & $\begin{array}{l}\text { Sim. Entre 25\% e 50\% das nossas etapas de produção possuem máquinas com } \\
\text { capacidade de ajustar a operação de acordo com informações recebidas de outras } \\
\text { máquinas. }\end{array}$ \\
\hline max pts. 100 & $\begin{array}{l}\text { O sistema de produção da nossa empresa permite que máquinas se comuniquem e se } \\
\text { ajustem de forma autônoma a requisitos de produção customizados e parâmetros } \\
\text { técnicos monitorados a fim de otimizar a eficácia operacional. }\end{array}$ \\
\hline
\end{tabular}

Fonte: os autores.

\section{CONCLUSÃO}

A evolução e convergência de tecnologias digitais e industriais sob o conceito da 14.0 vão revolucionar a maneira como bens são produzidos inclusive na pré-fabricação de componentes para sistemas construtivos industrializados. Esse artigo marca uma etapa intermediária de um trabalho de pesquisa de mestrado. Sua principal contribuição é a configuração das tecnologias da 14.0 para $\mathrm{FSCls}$ focado nas atividades de projeto e produção e o desenvolvimento de um MM direcionado às particularidades do setor.

Os próximos passos na pesquisa incluem a atribuição de pesos às questões do MMFSCl4.0, a validação do mesmo a fim de demonstrar a sua a confiabilidade como ferramenta de diagnóstico. Por fim, a coleta de dados de número significativo de fabricantes poderá traçar o retrato do estado atual da indústria e proporcionar um meio para definir estratégias para dar continuidade à industrialização tanto das empresas individuais quanto do setor dos $\mathrm{FSCls}$.

\section{REFERÊNCIAS}

[1] SABBATINI, Fernando H. Desenvolvimento de Métodos, Processos e Sistemas Construtivos: Formulação e Aplicação de uma Metodologia. 1989. Tese (Doutorado em Engenharia) - Escola Politécnica, Universidade de São Paulo, São Paulo, 1989.

[2] STÄIB, G.; DÖRRHÖFER, A.; ROSENTHAL, M. Components and Systems. Modular Construction Design, Structure, New Technologies. Munique: Birkhäuser, 2008.

[3] RAZKENARI, M.; FENNER, A.; SHOJAEI, A.; HAKIM, H. KIBERT, C. Perceptions of offsite construction in the United States: An investigation of current practices. Journal of Building Engineering, v. 29, maio, 2020. DOI: https://doi.org/10.1016/j.jobe.2019.101138

[4] MCKINSEY \& COMPANY. Modular construction: From projects to products. junho, 2019. 
[5] KAGERMANN, H.; LUKAS, W. WAHLSTER, W. Industrie 4.0: Mit dem Internet der Dinge auf dem Weg zur 4. industriellen Revolution. VDI nachrichten, n. 13. 2011.

[6] KAGERMANN, H.; LUKAS, W. WAHLSTER, W. HELBIG, J. Recommendations for implementing the strategic initiative Industrie 4.0: Final report of the Industrie 4.0 Working Group. 2013.

[7] DRESCH, A.; LACERDA, D.; ANTUNES JÚNIOR, A. Design Science Research: método de pesquisa para avanço da ciência e tecnologia. São Paulo: Bookman, 2015.

[8] ASSOCIAÇÃO BRASILEIRA DE NORMAS TÉCNICAS. NBR 15965-1: Sistema de classificação da informação da construção. Parte 1: Terminologia e estrutura, 2011.

[9] YIN, X.; LIU, H.; CHEN, Y.; AL-HUSSEIN, M. Building information modelling for off-site construction: Review and future directions. Automation in Construction, v. 101, p. 72-91, 2019. DOI: https://doi.org/10.1016/j.autcon.2019.01.010

[10] ABANDA, F. H.; TAH, J. H. M.; CHEUNG, F. K. T. BIM in off-site manufacturing for buildings. Journal of Building Engineering, v.14, n. 89-102, 2017. DOI: https://doi.org/10.1016/j.jobe.2017.10.002

[11] BRELL-COKCAN, S. et al. Zukunft Robotik - Automatisierungspotentiale im Stahl- und Metallleichtbau, Ernst \& Sohn Verlag für Architektur und technische Wissenschaften GmbH \& Co. KG, Berlin, Stahlbau 86, Heft 3, p. 225-233, 2017.

[12] LEE, J-S.; KWON, N.; HAM, N-H.; KIM, J-J.; AHN, Y-H. BIM-Based Digital Fabrication Process for a Free-Form Building Project in South Korea. Hindawi. Advances in Civil Engineering, v. 2019, 2019. DOI: https://doi.org/10.1155/2019/4163625

[13] ORLOWSKI, K. Automated manufacturing for timber-based panelised wall systems. Automation in Construction, v. 109, 102969, 2020. DOI: https://doi.org/10.1016/j.autcon.2019.102988

[14] PAN, M.; PAN, W. Determinants of Adoption of Robotics in Precast Concrete Production for Buildings. Journal of Management in Engineering, v. 35, n. 5, set. 2019. DOI: https://doi.org/10.1061/(ASCE)ME.1943-5479.0000706

[15] BOCK, T.; LINNER, T. Robotic industrialization: automation and robotic technologies for customized component, module and building prefabrication. New York: Cambridge University Press, 2015.

[16] GEISBERGER, E.; CENGARLE, M. V.; KEIL, P.; NIEHAUS, J.; THIEL, C.; THÖNNIßEN-FRIES, H.J.; Cyber-Physical Systems: Driving force for innovation in mobility, health, energy and production. ACATECH - National Academy Of Science And Engineering. 2011.

[17] LEE, E. A. Cyber Physical Systems: Design Challenges. In: SYMPOSIUM ON OBJECT ORIENTED REAL-TIME DISTRIBUTED COMPUTING, 11, 2008, Orlando. Proceedings [...]. New York: IEEE. p. 363-369. 2008. DOI: https://doi.org/10.1109/ISORC.2008.25

[18] LEE, E. A.; SESHIA, S. A. Introduction to Embedded Systems: A Cyber-Physical Systems Approach. ed. 2. MIT Press, 2017.

[19] MARWEDEL, P. Embedded System Design: Embedded Systems Foundations of CyberPhysical Systems, and the Internet of Things. ed. 4. Springer, 2021.

[20] MACHADO, Fernanda Almeida. BIM e Internet das Coisas para o monitoramento do consumo de energia da edificação. 2018. 208 p. Dissertação (mestrado) - Universidade Estadual de Campinas, Faculdade de Engenharia Civil, Arquitetura e Urbanismo, Campinas, SP.

[21] TANG, S.; et al. A review of BIM and the IoT devices integration: Present status and future trends. Automation in Construction, v. 101, p. 127-139, 2019. DOI: https://doi.org/10.1016/j.autcon.2019.01.020 
[22] BADGER, L.; GRANCE, T.; PARTT-CORNER, R.; VOAS, J. Cloud Computing Synopsis and Recommendations. National Institute of Standards and Technology, U.S. Department of Commerce, Special Publication 800-146. 2012.

[23] LIN, C.; RAMAKRISHNAN, K.; LIU, J.; NGAI, E. Guest Editorial Special Issue on Cloud Computing for loT. IEEE Internet of Things Journal, v. 3, n. 3, p. 254-256, 2016. DOI: https://doi.org/10.1109/JIOT.2016.2554738

[24] Grieves M., Vickers J. (2017) Digital Twin: Mitigating Unpredictable, Undesirable Emergent Behavior in Complex Systems. In: Kahlen FJ., Flumerfelt S., Alves A. (eds) Transdisciplinary Perspectives on Complex Systems. Springer, 2017. DOI: 10.1007/978-3319-38756-7_4

[25] SÖDERBERG, R.; WÄRMEFJORD, K.; CARLSON, J.S.; LINDKVIST, L. Toward a digital twin for real-time geometry assurance in individualized production. CIRP Annals, v. 66, n1., p.137-140, 2017. DOI: https://doi.org/10.1016/j.cirp.2017.04.038

[26] Negri, E., Fumagalli, L., Macchi, M. A review of the roles of digital twin in CPS-based production systems. Procedia Manufacturing. v.11, p. 939-948, 2017. DOI: https://doi.org/10.1016/j.promfg.2017.07.198

[27] GLAESSGEN, E.; STARGEL, D. The digital twin paradigm for future NASA and US Air Force vehicles. In: AIAA/ASME/ASCE/ AHS/ASC STRUCTURES, STRUCTURAL DYNAMICS AND MATERIALS CONFERENCE, 53., 2012, Honolulu. Proceedings [...]. Honolulu: AIAA, 2012. DOI: https://doi.org/10.2514/6.2012-1818

[28] BOSCHERT, S.; HEINRICH, C.; ROSEN, R. Next generation digital twin. In: INTERNATIONAL SYMPOSIUM ON TOOLS AND METHODS OF COMPETITIVE ENGINEERING, 12., 2018, Las Palmas de Gran Canaria. Proceedings [...]. Delft University of Technology: TMCE, 2018. p. 209-218.

[29] TAO, F.; QI, Q.; WANG, L. Digital twins and cyber-physical systems toward smart manufacturing and Industry 4.0: correlation and comparison, Engineering v. 5, p. $653-$ 661, 2019. DOI: https://doi.org/10.1016/j.eng.2019.01.014

[30] WAGIRE, A.; JOSHI, R.; RATHORE, A.; JAIN, R. Development of maturity model for assessing the implementation of Industry 4.0: learning from theory and practice. Production Planning \& Control, v. 32, n. 8, 2020. DOI: https://doi.org/10.1080/09537287.2020.1744763

[31] ROCKWELL AUTOMATION. The connected enterprise maturity model. Disponível em: <http://literature.rockwellautomation.com/idc/groups/literature/documents/wp/ciewp002_-en-p.pdf>. Acesso em: 12 de novembro, 2020.

[32] SCHUH, G.; ANDERL, R.; GAUSEMEIER, J.; ten HOMPEL, M.; WAHLSTER, W. Industrie 4.0 Maturity Index - Managing the Digital Transformation of Companies (acatech STUDY), Munich: Herbert Utz Verlag 2017.

[33] RWTH AACHEN UNIVERSITY. Industrie 4.0 in der Zementindustrie - Status quo und Perspektiven. Eine Studie im Auftrag der Initiative für Nachhaltigkeit in der deutschen Zementindustrie. 2019.

[34] LICHTBLAU, K.; STICH, V.; BERTENRATH, R.; BLUM, M.; BLEIDER, M.; MILLACK, A.; SCHMITT, K.; SCHMITZ, E.; SCHRÖTER, M. Industrie 4.0- Readiness. Aachen, Köln: ImpulsStiftung des VDMA. 2015.

[35] PwC - PricewaterhouseCoopers. The Industry 4.0 Digital Operations Self Assessment. 2016. Disponível em: <https://www.pwc.com/gx/en/industries/industries-4.0/landingpage/industry-4.0-building-yourdigital-enterprise-april-2016.pdf>. Acesso em: maio, 2021.

[36] SUCCAR, B. Building information modelling framework: a research and delivery foundation for industry stakeholders. Automation in Construction, v. 18 n. 3, p.357-375, 2009. DOI: https://doi.org/10.1016/j.autcon.2008.10.003 
[37] KAM, C.; SENARATNA, D.; MCKINNEY, B.; XIAO, Y.; SONG, M. The VDC Scorecard: Formulation and Validation. CIFE Working Paper \#WP135. Stanford University, 2013 